\title{
Novel screening model of obstructive sleep apnea for snorers with suspected NAFLD undergoing liver sonography
}

\author{
Yang-Bor Lu ${ }^{1,2}$, Yu-Chieh Weng ${ }^{1,2}$, Yung-Ning Huang ${ }^{1,2}$, Hsiung-Ying Huang ${ }^{3}$, Pei-Ting Cheng ${ }^{4}$, \\ Hui-Shan Hsieh ${ }^{5^{*}}$ and Ming-Shao Tsai ${ }^{6,7^{*}}$
}

\begin{abstract}
Background/aims: Given the increased incidence of obstructive sleep apnea (OSA) among patients with nonalcoholic fatty liver disease (NAFLD), noninvasive screening methods are urgently needed to screen for OSA risk in these patients when conducting an office-based assessment of hepatic steatosis. Therefore, we investigated the controlled attenuation parameter (CAP) and hepatic steatosis index (HSI) in patients with and without OSA and developed screening models to detect OSA.
\end{abstract}

Methods: We retrospectively reviewed the medical records of all adult snorers with suspected NAFLD undergoing liver sonography between June 2017 and June 2020. Records encompassed CAP and HSI data as well as data collected during in-hospital full-night polysomnography. The multivariate logistic regression models were constructed to explore the predictors of OSA risk. Furthermore, model validation was performed based on the medical records corresponding to the July 2020-June 2021 period.

Results: A total of 59 patients were included: $81.4 \%$ (48/59) were men, and the mean body mass index (BMI) was $26.4 \mathrm{~kg} / \mathrm{m}^{2}$. Among the patients, $62.7 \%$ (37/59) and 74.6\% (44/59) (detected by the HSI and CAP, respectively) had NAFLD, and 78\% (46/59) were diagnosed with OSA on the basis of polysomnography. Three screening models based on multivariate analysis were established. The model combining male sex, a BMI of $>24.8$, and an $\mathrm{HSI}$ of $>38.3$ screened for OSA risk the most accurately, with an area under the receiver operating characteristic curve of 0.81 (sensitivity: 78\%; specificity: $85 \%$; and positive and negative predictive values: $95 \%$ and $52 \%$, respectively) in the modeling cohort. An accuracy of $70.0 \%$ was achieved in the validation group.

Conclusions: The combination screening models proposed herein provide a convenient, noninvasive, and rapid screening tool for OSA risk and can be employed while patients receive routine hepatic check-ups. These models can assist physicians in identifying at-risk OSA patients and thus facilitate earlier detection and timely treatment initiation.

Keywords: Obstructive sleep apnea, Elastography, Fatty liver, Steatosis, Screen

*Correspondence: hsieh1111@gmail.com; b87401061@cgmh.org.tw ${ }^{5}$ Department of Otolaryngology-Head and Neck Surgery, Sleep Center, Xiamen Chang Gung Hospital, No. 123 Avenue Xiafei, Haicang District, Xiamen 361028, Fujian, China

${ }^{6}$ Department of Otolaryngology-Head and Neck Surgery, Chiayi Chang Gung Memorial Hospital, No. 6, Sec. W., Jiapu Rd., Puzi City 613, Chiayi County, Taiwan

Full list of author information is available at the end of the article

\section{Introduction}

Obstructive sleep apnea (OSA) is common, with a prevalence of $2 \%-4 \%$ in the general population and $35-45 \%$ in those who are obese [1]. In OSA, the upper airway collapses during sleep, which leads to snoring, hypercapnia, and complications related to chronic intermittent hypoxemia $(\mathrm{CIH})[2,3]$. OSA is associated with various 
metabolic dysfunctions, including insulin resistance and visceral obesity $[4,5]$. $\mathrm{CIH}$ is independently related to dyslipidemia in nonalcoholic fatty liver disease (NAFLD) $[6,7]$, thus exacerbating NAFLD and advancing liver fibrosis [8].

NAFLD affects one in four people worldwide [9] and comprises a broad spectrum of liver disorders. The obesity epidemic has increased the incidence of NAFLD [10] and the risk of OSA [11]. The prevalence of NAFLD is thus higher in patients with OSA [12]. Accordingly, it is crucial to screen patients with OSA risk for NAFLD to detect the condition at an early stage.

Noninvasive screening tools, including clinical laboratory parameters and transient elastography devices, have been developed to stage liver disease [13, 14]. One study performed transient elastography by using a novel proprietary algorithm called the controlled attenuation parameter (CAP), which was developed to quantify ultrasound attenuation during liver stiffness measurement (LSM) [15]. The CAP provides a numerical value that correlates with the histologic degree of steatosis [16] and has a favorable diagnostic value for chronic liver diseases $[17,18]$. The hepatic steatosis index (HSI), calculated on the basis of the serum aspartate aminotransferase (AST)/ alanine aminotransferase (ALT) ratio, body mass index (BMI), sex, and presence of diabetes mellitus, has been validated in a large cohort of more than 10,000 patients [19] and adopted as a screening method for NAFLD [20, 21].

Although patients with OSA and metabolic comorbidities may have higher LSM values [22], according to a review of the literature, no study has comprehensively compared the CAP and HSI values between individuals with and without OSA. This study investigated the performance of two NAFLD indices, the CAP and HSI, when applied to patients with and without OSA and developed models for OSA risk screening.

\section{Materials and methods}

\section{Study design and data source}

We retrospectively reviewed medical records from the Department of Digestive Disease at Xiamen Chang Gung Hospital between June 2017 and June 2020. All adult patients (aged $\geq 18$ years) with suspected NAFLD who underwent liver sonography and had self-reported snoring with in-hospital full-night polysomnography were identified. To prevent interference in the liver sonography and polysomnography results, we excluded the following individuals: (a) patients who had previously been diagnosed as having OSA for which they had received interventions (surgical or nonsurgical), including continuous positive airway pressure or oral appliance treatment, (b) patients with a history of excessive alcohol consumption (more than $30 \mathrm{~g} /$ day for men and more than $20 \mathrm{~g} /$ day for women), (c) and patients with a history of viral hepatitis or hepatic cancer [23]. The study protocol was approved by the Institutional Review Board of Xiamen Chang Gung Hospital (approval No. XMCGIRB 2021017), which waived the requirement for informed consent due to the retrospective nature of the study. All procedures were conducted in accordance with the current regulations.

\section{Anthropometric and laboratory evaluation}

Demographic data, such as age, sex, medical history, daily alcohol consumption, and drug usage, were obtained from medical chart review. Patients' comorbidities, including diabetes mellitus (DM) and hypertension, were also recorded. BMI was calculated as weight in $\mathrm{kg} /$ (height in $\mathrm{m})^{2}$, and waist circumference $(\mathrm{cm})$ was measured using a nonelastic measuring tape at the time of overnight polysomnography. The assessment of all laboratory parameters required for HSI calculation, including AST and ALT, was performed on the day of FibroScan or in the preceding week. The HSI was calculated automatically according to the following formula: $8 \times(\mathrm{ALT} /$ AST) + BMI (+2 if female; +2 if DM); HSI $>36.0$ has been validated as an indication of NAFLD, with a specificity of $92.4 \%$ [19].

\section{Polysomnography}

OSA parameters were documented for each patient during in-hospital full-night polysomnography (N7000 Embla, Broomfield, CO, USA). Polysomnography was scored manually by independent investigators according to the standard criteria [24]. The apnea-hypopnea index (AHI) was defined as the sum of all obstructive and mixed apnea ( $\geq 90 \%$ reduction in airflow for a duration of $\geq 2$ breaths) plus hypopnea ( $\geq 50 \%$ reduction in airflow, accompanied by $\geq 3 \%$ desaturation or electroencephalographic arousal, for a duration of $\geq 2$ breaths) divided by the number of hours of total sleep time and the oxygen desaturation index (ODI), which represents the number of events per hour of sleep with $\geq 3 \%$ desaturation from baseline [24]. The minimal $\mathrm{O}_{2}$ saturation (minimal $\mathrm{SpO}_{2}$ ), mean oxygen saturation $\left(\right.$ mean $\mathrm{SpO}_{2}$ ), and percentage of total recording time (\%TRT) with $\mathrm{SpO}_{2}<90 \%$ (T90\%) were also recorded. OSA was defined as $\mathrm{AHI} \geq 5$, and the patients with $\mathrm{AHI}<5$ were included in the non-OSA subgroup.

\section{CAP}

The FibroScan402 equipped with the standard M probe (Echosens, Paris, France) was operated by specially trained nurses according to normal procedures [25] to capture both CAP $(\mathrm{dB} / \mathrm{m})$ and LSM $(\mathrm{kPa})$ values 
simultaneously under fasting conditions for all patients. Examinations with poor reliability, defined as $<10$ valid acquisitions or a ratio of the interquartile range (IQR) over the median of 10 measurements (IQR/M) of LSM and CAP $>0.3$, were excluded [26]. On the basis of a previous cohort study, a CAP cutoff value of $\geq 238 \mathrm{dm} / \mathrm{m}$ was used to determine steatosis grade $\geq S 1$ ( $\geq 11 \%$ steatosis) [27].

\section{Validation}

Specifically, we extracted medical records from the Department of Digestive Disease corresponding to the July 2020-June 2021 period to perform model validation according to the consistent inclusion and exclusion criteria described previously.

\section{Statistical analysis}

All statistical analyses were completed using SAS version 9.4 (SAS Institute, Cary, North Carolina, USA). Because the preliminary Shapiro-Wilk normality test demonstrated that most variables did not follow a normal distribution, they were presented as the median and IQR and were compared using the Mann-Whitney U test. The chi-square test was used to compare categorical variables between the two groups. Correlations among hepatic indices, BMI, and other OSA severity markers were evaluated using the Spearman correlation. Variables with significant correlations were further subjected to logistic regression analysis. The results of univariate logistic analysis exploring predictors of OSA risk are expressed as odds ratios (ORs) with 95\% confidence intervals (CIs). The results of multivariate logistic analysis exploring predictors of OSA risk are expressed as adjusted ORs with 95\% CIs. Given that the calculation of HSI takes into account sex, BMI, ALT, AST, and diabetes, sex and BMI may be potential confounders. We further subjected the confounding factors to multivariate logistic regression with the interaction terms HSI $\times$ sex and HSI $\times$ BMI and found no significant effects. Thus, the confounding effects were not considered in the subsequent combined models. Subsequently, variables with $p$ values of $<0.05$ in the logistic regression analysis were dichotomized according to the optimal cutoff value by using receiver operating characteristic (ROC) curves [28]. The dichotomized variables were then assessed using multivariate logistic regression to construct combined models. The sensitivity, specificity, positive predictive value (PPV), and negative predictive value (NPV) for each dichotomized value of the predictors and combined models were calculated. The most commonly used index of accuracy was the area under the ROC curve (AUC). The ROC curves were constructed by plotting the sensitivity against ( $1-$ specificity) for each combined model. All $p$ values were two-sided, and statistical significance was represented by $p<0.05$.

\section{Results}

Patients' characteristics in the modeling group

A total of 94 adult patients with suspected NAFLD and undergoing liver sonography as well as polysomnography were identified. After 35 patients were excluded from the analysis (4 patients had previously been diagnosed as having OSA, 14 patients had a history of excessive alcohol consumption, and 17 patients had a history of viral hepatitis or hepatic cancer), 59 patients were finally enrolled. After the polysomnography reports and medical records of these 59 patients were reviewed, the 46 patients with confirmed OSA $(\mathrm{AHI} \geq 5)$ were designated as the OSA group, and the 13 patients with an AHI of $<5$ were designated as the non-OSA group. The patients' characteristics are presented in Table 1. The OSA group had a male predominance, a significantly higher BMI $(p<0.01)$, and a significantly greater HSI $(p=0.03)$. Moreover, as expected, it had a significantly lower mean $\mathrm{SpO}_{2}$, minimal $\mathrm{SpO}_{2}$, ODI, and T90(\%) (all $p<0.01$ ). No significant between-cohort differences in CAP value $(p=0.67)$ or other hepatic indices were observed.

\section{Correlations between hepatic indices, BMI, and OSA severity parameters}

Significant correlations were observed between the CAP and the following OSA severity indices: AHI $(r=0.35$, $p<0.01)$, mean $\mathrm{SpO}_{2}(r=-0.35, p<0.01)$, minimal $\mathrm{SpO}_{2}$ $(r=-0.29, p<0.05), \mathrm{ODI} \geq 3 \%$ (ODI3; $r=0.33, p<0.01$ ), and T90\% $(r=0.39, p<0.01$; Table 2$)$. The HSI was also significantly associated with AHI $(r=0.33, p<0.05)$ and ODI3 $(r=0.26, p<0.05)$. No other liver parameters had significant correlations with OSA severity indices. BMI was also significantly associated with OSA severity parameters.

\section{Predictors and screening models for OSA risk}

From the screening results, we identified predictors of OSA risk (Table 3). Univariate logistic regression revealed that male sex (OR 4.17, 95\% CI 1.02-17.05), $\mathrm{BMI}>24.8$ (OR 1.42, 95\% CI 1.09-1.86), and HSI > 38.3 (OR 1.17, 95\% CI 1.02-1.36) are significant factors in screening for OSA risk, whereas multivariate logistic regression analysis revealed that male sex (adjusted OR 22.92, 95\% CI 1.76-298.92), BMI $>24.8$ (adjusted OR 1.83, 95\% CI 1.07-3.11), and CAP > 243.5 (adjusted OR 0.96 , 95\% CI 0.93-0.99) are independent significant predictors of OSA risk. Regarding sex and BMI, the factors with potential confounding effects on HSI, no significant confounding effect was noted in the multivariate logistic regression $(p=0.77$ and 0.11 , 
Table 1 Baseline clinicodemographic characteristics of the modeling cohort

\begin{tabular}{|c|c|c|c|c|}
\hline & All & Non-OSA group & OSA group & $p$ value \\
\hline Patients (n) & 59 & 13 & 46 & \\
\hline Sex, Males/female & $48(81.4) / 11(18.6)$ & $8(61.5) / 5(38.5)$ & $40(87.0) / 6(13.0)$ & 0.04 \\
\hline Age (years) & $44.3 \pm 8.59$ & $42.1 \pm 6.78$ & $44.9 \pm 9.01$ & 0.30 \\
\hline $\mathrm{BMI}\left(\mathrm{kg} / \mathrm{m}^{2}\right)$ & $26.4 \pm 3.0$ & $24.4 \pm 3.0$ & $27.0 \pm 2.7$ & $<0.01$ \\
\hline Waist circumference $(\mathrm{cm})$ & $86.3 \pm 8.8$ & $82.1 \pm 6.6$ & $87.4 \pm 9.0$ & 0.05 \\
\hline Diabetes & $4(6.8)$ & $0(0.0)$ & $4(8.7)$ & 0.27 \\
\hline Hypertension & $14(23.7)$ & $2(15.4)$ & $12(26.1)$ & 0.42 \\
\hline AHI (events/h) & $16.7(5.9-42.2)$ & $1.7(0.6-3.0)$ & $23.9(11.3-49.0)$ & $<0.01$ \\
\hline Mean $\mathrm{SpO}_{2}(\%)$ & $96.1(94.0-96.5)$ & $96.5(96.2-97.0)$ & $95.8(93.9-96.4)$ & $<0.01$ \\
\hline Minimal $\mathrm{SpO}_{2}(\%)$ & $87.0(80.0-92.0)$ & $93.0(92.0-94.0)$ & $85.0(75.0-90.0)$ & $<0.01$ \\
\hline ODI (events/h) & $12.3(2.0-29.5)$ & $0.6(0.0-1.4)$ & $20.1(8.4-43.3)$ & $<0.01$ \\
\hline T90 (\%) & $0.1(0.0-1.9)$ & $0.0(0.0-0.0)$ & $0.3(0.0-2.9)$ & $<0.01$ \\
\hline $\operatorname{ALT}(\mathrm{IU} / \mathrm{L})$ & $32.0(21.0-44.0)$ & $23.0(18.0-32.0)$ & $34.0(22.0-48.0)$ & 0.09 \\
\hline AST (IU/L) & $26.0(20.0-32.0)$ & $24.0(21.0-27.0)$ & $27.0(20.0-34.0)$ & 0.17 \\
\hline $\mathrm{CAP}(\mathrm{dB} / \mathrm{m})$ & $269.4 \pm 46.4$ & $264.5 \pm 47.8$ & $270.8 \pm 46.4$ & 0.67 \\
\hline Median & $262.0(236.0-310.0)$ & $243.0(240.0-304.0)$ & $262.5(235.0-310.0)$ & 0.71 \\
\hline$\geq 238$ & $44(74.6 \%)$ & 10 (76.9\%) & $34(73.9 \%)$ & 0.83 \\
\hline $\mathrm{HSI}$ & $37.4 \pm 6.1$ & $34.2 \pm 4.5$ & $38.3 \pm 6.2$ & 0.03 \\
\hline Median & $37.5(33.1-41.0)$ & $33.1(32.1-37.1)$ & $38.2(34.3-41.3)$ & 0.02 \\
\hline$>36$ & $37(62.7 \%)$ & $6(46.2 \%)$ & $31(67.4 \%)$ & 0.16 \\
\hline LSM (kPa) & $5.3(4.5-6.2)$ & $4.9(4.1-5.7)$ & $5.6(4.6-6.7)$ & 0.10 \\
\hline
\end{tabular}

AHI apnea-hypopnea index, ALT alanine aminotransferase, AST aspartate aminotransferase, BMI body mass index, CAP controlled attenuation parameter, HSI hepatic steatosis index, LSM liver stiffness measurement, $\mathrm{ODI}$ oxygen desaturation index, $\mathrm{OSA}$ obstructive sleep apnea, $\mathrm{SpO}_{2}$ oxygen saturation measured by pulse oximetry, T90 the percentage of total recording sleep time with oxygen saturation $<90 \%$

Data are summarized as mean \pm standard deviation, median (interquartile range), or $\mathrm{n}(\%)$. Data were compared between non-OSA and OSA groups using the MannWhitney $\mathrm{U}$ test or chi-square test. Significant $p$ values are marked in bold

Table 2 Correlations among hepatic indices, BMI, and OSA severity parameters

\begin{tabular}{|c|c|c|c|c|c|c|c|c|c|c|}
\hline & \multicolumn{2}{|l|}{$\mathrm{AHI}$} & \multicolumn{2}{|c|}{ Mean $\mathrm{SpO}_{2}$} & \multicolumn{2}{|c|}{ Minimal $\mathrm{SpO}_{2}$} & \multicolumn{2}{|l|}{ ODI3 } & \multicolumn{2}{|l|}{ T90\% } \\
\hline & $r$ & $P$ & $r$ & $p$ & $r$ & $p$ & $r$ & $p$ & $r$ & $p$ \\
\hline ALT & 0.16 & 0.24 & -0.03 & 0.85 & -0.02 & 0.90 & 0.02 & 0.89 & 0.03 & 0.84 \\
\hline AST & 0.09 & 0.51 & 0.05 & 0.71 & 0.07 & 0.60 & -0.04 & 0.78 & -0.07 & 0.57 \\
\hline $\mathrm{BMl}$ & 0.45 & $<0.01$ & -0.34 & $<0.01$ & -0.29 & $<0.05$ & 0.40 & $<0.01$ & 0.27 & $<0.05$ \\
\hline CAP & 0.35 & $<0.01$ & -0.35 & $<0.01$ & -0.29 & $<0.05$ & 0.33 & $<0.01$ & 0.39 & $<0.01$ \\
\hline $\mathrm{HSI}$ & 0.33 & $<0.05$ & -0.22 & 0.10 & -0.19 & 0.15 & 0.26 & $<0.05$ & 0.18 & 0.18 \\
\hline
\end{tabular}

The correlations among the hepatic indices, BMI, and other OSA severity parameters were evaluated using the Spearman correlation. Significant $p$ values are marked in bold

AHI apnea-hypopnea index, $A L T$ alanine aminotransferase, $A S T$ aspartate aminotransferase, $B M I$ body mass index, CAP controlled attenuation parameter, HSI hepatic steatosis index, $\mathrm{ODI}$ oxygen desaturation index, OSA obstructive sleep apnea, $\mathrm{SpO}_{2}$ oxygen saturation measured by pulse oximetry; $\mathrm{T} 90 \%$, the percentage of total recording time with oxygen saturation $<90 \%$

respectively). In the subsequent ROC curve analysis, male sex, BMI, the CAP, and the HSI demonstrated fair to acceptable diagnostic performance in screening for OSA risk, with the AUC ranging from 0.54 to 0.75 (Table 4). By using multivariate logistic regression analysis, we constructed the following screening models for OSA risk.
Combined model A included three or more of the following predictors of OSA risk: male sex, $\mathrm{BMI}>24.8$, CAP > 243.5, and HSI > 38.3; it achieved an AUC of 0.75 (Fig. 1), with a sensitivity of $65 \%$, a specificity of $85 \%$, a PPV of $94 \%$, and NPV of $41 \%$.

Combined model B included two or more of the following predictors of OSA risk: male sex, BMI $>24.8$, 
Table 3 NAFLD-related parameters derived from the OSA risk screening

\begin{tabular}{|c|c|c|c|c|}
\hline & \multicolumn{2}{|l|}{ Univariate } & \multicolumn{2}{|l|}{ Multivariate } \\
\hline & OR (95\% Cl) & $p$ value & $\begin{array}{l}\text { Adjusted OR (95\% } \\
\text { Cl) }\end{array}$ & $p$ value \\
\hline Male & $4.17(1.02-17.05)$ & 0.04 & $22.92(1.76-298.92)$ & 0.02 \\
\hline Age $>47.5$ & $1.04(0.96-1.35)$ & 0.29 & $1.16(1.00-1.35)$ & 0.05 \\
\hline $\mathrm{BMI}>24.8$ & $1.42(1.09-1.86)$ & 0.01 & $1.83(1.07-3.11)$ & 0.03 \\
\hline CAP $>243.5$ & $1.00(0.99-1.02)$ & 0.66 & $0.96(0.93-0.99)$ & 0.02 \\
\hline $\mathrm{HSI}>38.3$ & $1.17(1.02-1.36)$ & 0.03 & $1.33(0.98-1.80)$ & 0.07 \\
\hline
\end{tabular}

Significant $p$ values are marked in bold

$B M I$ body mass index, $C A P$ controlled attenuation parameter, $C l$ confidence interval, $H S$ I hepatic steatosis index, OR odds ratio

and CAP > 243.5; it achieved an AUC of 0.71, with a sensitivity of $80 \%$, a specificity of $62 \%$, a PPV of $88 \%$, and NPV of $47 \%$.

Combined model $\mathrm{C}$ included two or more of the following predictors of OSA risk: male sex, BMI $>24.8$, and HSI > 38.3; it achieved an AUC of 0.81, with a sensitivity of $78 \%$, a specificity of $85 \%$, a PPV of $95 \%$, and NPV of $52 \%$.

\section{Validation of the screening model for OSA risk}

Given the availability of in-hospital polysomnography studies has decreased amid the ongoing COVID-19 pandemic [29] and given some individuals at risk for OSA may not be identified, the sample of 29 patients, which was compared with our modeling cohort. The characteristics of the 29 patients are presented in the Additional file 1: Table S1 and compared with those of the modeling cohort (Additional file 1: Table S2). The screening accuracy of combined models $\mathrm{A}, \mathrm{B}$, and $\mathrm{C}$ in the validation group was $65.6 \%, 75.9 \%$, and $70.0 \%$, respectively.

\section{Discussion}

Studies have reported the mutually reinforcing pathogenic effects in NAFLD and OSA [30-32]. Our findings revealed that hepatic steatosis parameters are significantly related to OSA severity and hypoxic burdens, including AHI and ODI3. These findings accord with a previous report that identified a significant correlation between the CAP and the respiratory event index [33]. CIH is a causal factor of NAFLD progression, independent of obesity and metabolic syndrome [34], possibly owing to oxidative stress and mitochondrial dysfunction [35]. However, the roles of various transcription mechanisms of $\mathrm{CIH}$-induced hepatic steatosis remain unclear

Table 4 Screening models for OSA risk

\begin{tabular}{|c|c|c|c|c|c|c|c|c|}
\hline \multirow[t]{2}{*}{ Predictors } & \multicolumn{2}{|l|}{ Logistic regression } & \multicolumn{6}{|c|}{ Receiver operating characteristic curve } \\
\hline & OR $(95 \% \mathrm{Cl})$ & $p$ value & Cutoff value & sensitivity & specificity & PPV & NPV & AUC \\
\hline \multicolumn{9}{|l|}{ Original models } \\
\hline Male & $4.2(1.02-17.05)$ & 0.047 & Male & $87 \%$ & $39 \%$ & $83 \%$ & $46 \%$ & 0.63 \\
\hline BMI & $8.1(2.06-31.88)$ & $<0.01$ & $>24.8$ & $78 \%$ & $69 \%$ & $90 \%$ & $47 \%$ & 0.75 \\
\hline CAP & $2.4(0.69-8.44)$ & 0.17 & $>243.5$ & $67 \%$ & $54 \%$ & $84 \%$ & $32 \%$ & 0.54 \\
\hline $\mathrm{HSI}$ & $10.1(1.21-84.03)$ & 0.03 & $>38.3$ & $46 \%$ & $92 \%$ & $96 \%$ & $32 \%$ & 0.72 \\
\hline Combined models & Adjusted OR (95\% Cl) & $p$ value & Predictors & & & & & \\
\hline Model A & & & $\geq 3$ & $65 \%$ & $85 \%$ & $94 \%$ & $41 \%$ & 0.75 \\
\hline Male & $2.5(0.5-12.4)$ & 0.28 & & & & & & \\
\hline $\mathrm{BMI}>24.8$ & $5.3(1.1-25.6)$ & 0.04 & & & & & & \\
\hline$C A P>243.5$ & $0.6(0.1-3.0)$ & 0.54 & & & & & & \\
\hline $\mathrm{HSI}>38.3$ & $6.4(0.7-61.2)$ & 0.11 & & & & & & \\
\hline Model B & & & $\geq 2$ & $80 \%$ & $62 \%$ & $88 \%$ & $47 \%$ & 0.71 \\
\hline Male & $2.4(0.5-11.7)$ & 0.28 & & & & & & \\
\hline $\mathrm{BMI}>24.8$ & $7.0(1.5-33.6)$ & 0.02 & & & & & & \\
\hline$C A P>243.5$ & $0.9(0.2-4.2)$ & 0.87 & & & & & & \\
\hline Model C & & & $\geq 2$ & $78 \%$ & $85 \%$ & $95 \%$ & $52 \%$ & 0.81 \\
\hline Male & $2.3(0.5-11.1)$ & 0.30 & & & & & & \\
\hline $\mathrm{BMI}>24.8$ & $4.5(1.0-19.4)$ & 0.045 & & & & & & \\
\hline $\mathrm{HSI}>38.3$ & $5.6(0.6-51.9)$ & 0.13 & & & & & & \\
\hline
\end{tabular}

Significant $p$ values are marked in bold

$A U C$ area under the curve, $B M I$ body mass index, $C A P$ controlled attenuation parameter, $C l$ confidence interval, $H S I$ hepatic steatosis index, $N P V$ negative predictive value, $O R$ odds ratio, $P P V$ positive predictive value 


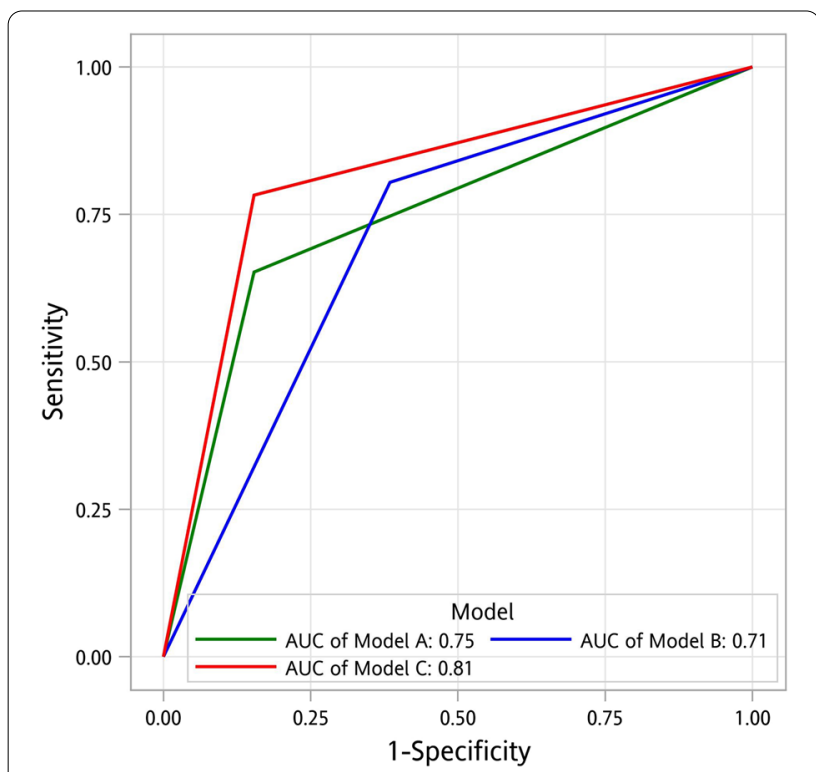

Fig. 1 Receiver operating characteristic (ROC) curves of the combined models in the modeling cohort. AUC area under the ROC curve

[33]. In the present study, AHI and ODI were both significant factors related to the CAP and HSI, whereas T90\% was not significantly related to the HSI. This finding implies that ODI, reflecting oxygen desaturation frequency, may be more specific for OSA-related $\mathrm{CIH}$ than T90\%, reflecting hypoxemia severity $[22,36]$.

Our study also identified the population at high risk for the co-occurrence of NAFLD and OSA. Among patients with NAFLD, $64 \%-87 \%$ have OSA [12, 37-39]; in our study, the prevalence of liver steatosis in the OSA cohort was $73.9 \%(34 / 46)$, a finding that warrants the development of an effective, reproducible, and noninvasive method of screening for OSA risk in this patient population. In the present study, the HSI and CAP both exhibited a high PPV for OSA ( $96 \%$ and $84 \%$, respectively). Furthermore, HSI $>38.3$ was more effective in screening for OSA risk than was CAP > 243.5 (AUC: 0.72 and 0.54, respectively), whereas the $C A P$ demonstrated higher sensitivity ( $67 \%$ vs. $46 \%)$. CAP $\geq 238 \mathrm{dm} / \mathrm{m}$ was reported to indicate hepatic steatosis ( $>10 \%$ fatty deposit) [27]; the cutoff value of the CAP to screen for OSA risk in our study was somewhat higher $(>243.5 \mathrm{dm} / \mathrm{m})$, implying that OSA worsens hepatic steatosis in individuals with concomitant OSA and NAFLD. HSI $>36.0$ was demonstrated to have a $92.4 \%$ specificity for detecting NAFLD [19]; in our study, HSI $>38.3$ had a $92 \%$ specificity for detecting OSA, and the AUC of screening for NAFLD in OSA was similar to that in a previous study [40], but that study reported a lower cutoff value of 35 [40]. This discrepancy might be due to differences in the severity of hepatic steatosis in each cohort. The increased HSI value may reflect the degree of hepatic steatosis, similar to liver echogenicity on ultrasound $[19,41]$. Moreover, the higher cutoff value of the HSI in our study might indicate the likelihood of severer steatosis when OSA and fatty liver coexist. Compared with the CAP or HSI alone, the accuracy of screening for OSA risk was higher in the combined models.

The CAP exhibited a lower AUC for screening OSA risk than the HSI not only in the original models (0.54 and 0.72 , respectively) but also in the combined models ( 0.71 and 0.81 , respectively), thereby implying that the HSI may be a more accurate tool for screening for OSA risk in individuals with concomitant OSA and NAFLD. The HSI formula uses liver function parameters and demographic characteristics, and thus, it reflects systemic status rather local hepatic elastography, as the CAP does; this approach suggests that OSA is not merely a local event but has further systemic effects [42]. The risk of developing OSA was determined to increase with age and BMI [43] and significant correlations among OSA severity parameters and BMI were also observed in the present study. The fact that BMI was considered in the HSI calculation may explain why the HSI was more accurate in predicting OSA risk in the screening than was the CAP. Although these two hepatic indices differ in screening performance, they are easier to access, provide quantitative results compared with semiquantitative ultrasonography [40], and demonstrate improved accuracy in combination models. The accuracy in the validation group may be affected by the sample size being only half that of the modeling cohort. Nevertheless, we contend that our findings can serve as a reference for clinicians to screen OSA risk in patients with NAFLD more efficiently. The office-based screening models presented in this study may help identify the subpopulation that is more likely to develop OSA before time-consuming examinations such as polysomnography are conducted, thus lowering clinical costs and workloads, especially in places with limited resources. Our simplified screening models use noninvasive and rapid tests that can easily screen patients for OSA risk while surveying hepatic steatosis; thus, they enable the prioritization of at-risk individuals for further assessment and early management. Furthermore, effective treatment of OSA, such as continuous positive airway pressure, can prevent hypoxic events related to upper airway collapse [44], which may stabilize or retard NAFLD progression $[38,45]$ and hence prevent more hazardous comorbidities.

Our study has some limitations. First, selection bias is possible because we included patients who initially presented to the outpatient clinic in the Department of Digestive Disease for routine check-ups with 
self-reported snoring. Moreover, we included patients who agreed to undergo in-hospital full-night polysomnography when their physician asked whether they snored. Given that the study cohort did not include patients with a history of hepatic cancer or viral hepatitis and that the combined models were based on NAFLDrelated parameters, our models are applicable to individuals with NAFLD. The findings are expected to enhance awareness of sleep apnea among physicians other than sleep specialists, such as hepatologists, who are likely to encounter patients at risk for OSA in daily clinical practice. This simple model can be readily applied in officebased to prioritize early intervention for at-risk patients. Second, liver biopsy data were lacking in this study, the reliability of the CAP varied depending on steatosis grade [46], and the HSI was based on ultrasonography only [19]. Third, our sample size was small; nevertheless, the predictors with AUCs exceeding 0.7 (BMI and HSI) reached a power of $\geq 70 \%$. Furthermore, combined model $C$ reached a power of $\geq 99 \%$ and had an acceptable accuracy in the validation cohort. For combined model $\mathrm{B}$, however, theoretically, at least 100 patients must be included to reach a power of $\geq 90 \%$. Further prospective research should employ a larger sample size to optimize the screening models for OSA.

\section{Conclusions}

Significant correlations were observed between the CAP, the HSI, and OSA severity. The HSI seems to be a more accurate indicator than the CAP for OSA risk screening. The models for OSA risk presented in this study may serve as screening tools for individuals receiving routine transabdominal elastography or fatty liver examination. The combined models are applicable to the development of precision medicine protocols for patients with NAFLD and OSA. Furthermore, because of the convenience, noninvasiveness, and rapidity of these screening models, patients suspected of having NAFLD can be screened for OSA risk, thereby facilitating early intervention and management.

\footnotetext{
Abbreviations

AHI: Apnea-hypopnea index; ALT: Alanine aminotransferase; AST: Aspartate aminotransferase; AUC: Area under the curve; BMI: Body mass index; CAP: Controlled attenuation parameter; Cl: Confidence interval; HSI: Hepatic steatosis index; LSM: Liver stiffness measurement; NAFLD: Nonalcoholic fatty liver disease; NPV: Negative predictive value; ODI: Oxygen desaturation index; OR: Odds ratio; OSA: Obstructive sleep apnea; PPV: Positive predictive value; $\mathrm{SpO}_{2}$ : Oxygen saturation measured by pulse oximetry; T90\%: The percentage of total recording sleep time with oxygen saturation $<90 \%$.
}

\section{Supplementary Information}

The online version contains supplementary material available at https://doi. org/10.1186/s12890-021-01759-1.

Additional file 1. Table S1: Baseline clinicodemographic characteristics of the validation cohort. Table S2: Baseline clinicodemographic characteristics of validation cohort and modeling cohort.

\section{Acknowledgements}

The manuscript was edited by Wallace Academic Editing.

\section{Authors' contributions}

Conceptualization, Y.B.L., Y.C.W., Y.N.H., and H.S.H.; Methodology, Y.B.L., and Y.C.W.; Formal analysis, Y.C.W., H.Y.H., P.T.C., and H.S.H.; Data curation P.T.C., H.S.H., and M.S.T; Writing-original draft preparation Y.B.L., P.T.C., and H.S.H.; Writing-review and editing, H.S.H, and M.S.T.; Visualization, P.T.C., and M.S.T.; Project administration, Y.B.L., and H.S.H. All authors read and approved the final manuscript

\section{Funding}

This research was supported by the Xiamen Municipal Science and Technology Bureau (Grant Number 3502Z20199156). The funder had no role in study design, data collection, and analysis, decision to publish, or preparation of the manuscript.

\section{Availability of data and materials}

All data supporting the finding of this study are available within the manuscript. The datasets generated and/or analyzed during the current study are available from the corresponding author upon reasonable written request.

\section{Declarations}

\section{Ethics approval and consent to participate}

This retrospective analysis utilized deidentified data from human subjects. This study approved by the Institutional Review Board of Xiamen Chang Gung hospital (No: XMCGIRB 2021017). Informed consent was waived because of the retrospective nature of the study and was approved by the Institutional Review Board of Xiamen Chang Gung hospital. Study was carried out in accordance with the Declaration of Helsinki. All methods were carried out in accordance with relevant guidelines and regulations in the Ethics approval.

\section{Consent for publication}

Not applicable.

\section{Competing interests}

The authors declare no competing interests.

\section{Author details \\ ${ }^{1}$ Department of Digestive Disease, Xiamen Chang Gung Hospital, Xiamen, China. ${ }^{2}$ Hepatobiliary and Pancreatic Unit, Xiamen Chang Gung Hospital, Xiamen, China. ${ }^{3}$ Department of Pulmonary and Critical Care Medicine, Sleep Center, Xiamen Chang Gung Hospital, Xiamen, China. ${ }^{4}$ Formosa Biomedi- cal Technology Corp., Taipei, Taiwan. ${ }^{5}$ Department of Otolaryngology-Head and Neck Surgery, Sleep Center, Xiamen Chang Gung Hospital, No. 123 Avenue Xiafei, Haicang District, Xiamen 361028, Fujian, China. ${ }^{6}$ Department of Otolaryngology-Head and Neck Surgery, Chiayi Chang Gung Memorial Hospital, No. 6, Sec. W., Jiapu Rd., Puzi City 613, Chiayi County, Taiwan. ${ }^{7}$ College of Medicine, Chang Gung University, Taoyuan, Taiwan.}

Received: 16 June 2021 Accepted: 22 November 2021

Published online: 26 November 2021 


\section{References}

1. Young T, Palta M, Dempsey J, Skatrud J, Weber S, Badr S. The occurrence of sleep-disordered breathing among middle-aged adults. N Engl J Med. 1993;328(17):1230-5.

2. Mannarino MR, Di Filippo F, Pirro M. Obstructive sleep apnea syndrome. Eur J Intern Med. 2012;23(7):586-93.

3. Lévy P, Kohler M, McNicholas WT, Barbé F, McEvoy RD, Somers VK, Lavie L, Pépin JL. Obstructive sleep apnoea syndrome. Nat Rev Dis Primers. 2015:1:15015.

4. Drager LF, Togeiro SM, Polotsky VY, Lorenzi-Filho G. Obstructive sleep apnea: a cardiometabolic risk in obesity and the metabolic syndrome. J Am Coll Cardiol. 2013;62(7):569-76.

5. Ip MS, Lam B, Ng MM, Lam WK, Tsang KW, Lam KS. Obstructive sleep apnea is independently associated with insulin resistance. Am J Respir Crit Care Med. 2002;165(5):670-6.

6. Song SO, He K, Narla RR, Kang HG, Ryu HU, Boyko EJ. Metabolic consequences of obstructive sleep apnea especially pertaining to diabetes mellitus and insulin sensitivity. Diabetes Metab J. 2019:43(2):144-55.

7. Adedayo AM, Olafiranye O, Smith D, Hill A, Zizi F, Brown C, Jean-Louis G. Obstructive sleep apnea and dyslipidemia: evidence and underlying mechanism. Sleep Breath. 2014;18(1):13-8.

8. Aron-Wisnewsky J, Clement K, Pépin JL. Nonalcoholic fatty liver disease and obstructive sleep apnea. Metabolism. 2016;65(8):1124-35.

9. Estes C, Razavi H, Loomba R, Younossi Z, Sanyal AJ. Modeling the epidemic of nonalcoholic fatty liver disease demonstrates an exponential increase in burden of disease. Hepatology. 2018;67(1):123-33

10. Ogden CL, Carroll MD, Flegal KM. Prevalence of obesity in the United States. JAMA. 2014;312(2):189-90.

11. Kopelman PG. Obesity as a medical problem. Nature 2000;404(6778):635-43

12. Trzepizur W, Boursier J, Mansour Y, Le Vaillant M, Chollet S, Pigeanne T, Bizieux-Thaminy A, Humeau MP, Alizon C, Goupil F, et al. Association between severity of obstructive sleep apnea and blood markers of liver injury. Clin Gastroenterol Hepatol. 2016;14(11):1657-61.

13. Asrani SK. Incorporation of noninvasive measures of liver fibrosis into clinical practice: diagnosis and prognosis. Clin Gastroenterol Hepatol. 2015;13(12):2190-204.

14. Machado MV, Cortez-Pinto H. Non-invasive diagnosis of non-alcoholic fatty liver disease. A critical appraisal. J Hepatol. 2013;58(5):1007-19.

15. Wong GL, Wong VW. Fat and fiber: how the controlled attenuation parameter complements noninvasive assessment of liver fibrosis. Dig Dis Sci. 2015:60(1):9-12.

16. Sasso M, Miette $V$, Sandrin L, Beaugrand M. The controlled attenuation parameter (CAP): a novel tool for the non-invasive evaluation of steatosis using Fibroscan. Clin Res Hepatol Gastroenterol. 2012;36(1):13-20.

17. Kumar M, Rastogi A, Singh T, Behari C, Gupta E, Garg H, Kumar R, Bhatia V, Sarin SK. Controlled attenuation parameter for non-invasive assessment of hepatic steatosis: does etiology affect performance? J Gastroenterol Hepatol. 2013;28(7):1194-201.

18. Myers RP, Pollett A, Kirsch R, Pomier-Layrargues G, Beaton M, Levstik M, Duarte-Rojo A, Wong D, Crotty P, Elkashab M. Controlled Attenuation Parameter (CAP): a noninvasive method for the detection of hepatic steatosis based on transient elastography. Liver Int. 2012;32(6):902-10.

19. Lee JH, Kim D, Kim HJ, Lee CH, Yang Jl, Kim W, Kim YJ, Yoon JH, Cho SH, Sung MW, et al. Hepatic steatosis index: a simple screening tool reflecting nonalcoholic fatty liver disease. Dig Liver Dis. 2010;42(7):503-8.

20. Sviklāne L, Olmane E, Dzērve Z, Kupčs K, Pīrāgs V, Sokolovska J. Fatty liver index and hepatic steatosis index for prediction of non-alcoholic fatty liver disease in type 1 diabetes. J Gastroenterol Hepatol. 2018;33(1):270-6.

21. Kahl S, Straßburger K, Nowotny B, Livingstone R, Klüppelholz B, Keßel K, Hwang JH, Giani G, Hoffmann B, Pacini G, et al. Comparison of liver fat indices for the diagnosis of hepatic steatosis and insulin resistance. PLoS ONE. 2014;9(4):e94059.

22. Trzepizur W, Boursier J, Le Vaillant M, Ducluzeau PH, Dubois S, Henni S, Abraham P, Aubé C, Calès P, Gagnadoux F. Increased liver stiffness in patients with severe sleep apnoea and metabolic comorbidities. Eur Respir J. 2018;51(6):66.

23. Burgos-Sanchez C, Jones NN, Avillion M, Gibson SJ, Patel JA, Neighbors J, Zaghi S, Camacho M. Impact of alcohol consumption on snoring and sleep Apnea: a systematic review and meta-analysis. Otolaryngol Head Neck Surg. 2020;163(6):1078-86.
24. Berry RB, Budhiraja R, Gottlieb DJ, Gozal D, Iber C, Kapur VK, Marcus CL, Mehra R, Parthasarathy S, Quan SF, et al. Rules for scoring respiratory events in sleep: update of the 2007 AASM manual for the scoring of sleep and associated events. Deliberations of the Sleep Apnea Definitions Task Force of the American Academy of Sleep Medicine. J Clin Sleep Med. 2012;8(5):597-619.

25. de Lédinghen V, Vergniol J. Transient elastography (FibroScan). Gastroenterol Clin Biol. 2008;32(6 Suppl 1):58-67.

26. Schwabl P, Bota S, Salzl P, Mandorfer M, Payer BA, Ferlitsch A, Stift J, Wrba F, Trauner M, Peck-Radosavljevic M, et al. New reliability criteria for transient elastography increase the number of accurate measurements for screening of cirrhosis and portal hypertension. Liver Int. 2015;35(2):381-90.

27. Sasso M, Beaugrand M, de Ledinghen V, Douvin C, Marcellin P, Poupon $\mathrm{R}$, Sandrin L, Miette V. Controlled attenuation parameter (CAP): a novel VCTE ${ }^{\mathrm{TM}}$ guided ultrasonic attenuation measurement for the evaluation of hepatic steatosis: preliminary study and validation in a cohort of patients with chronic liver disease from various causes. Ultrasound Med Biol. 2010;36(11):1825-35.

28. Reibnegger G, Schrabmair W. Optimum binary cut-off threshold of a diagnostic test: comparison of different methods using Monte Carlo technique. BMC Med Inform Decis Mak. 2014;14(1):99.

29. Miller MA, Cappuccio FP. A systematic review of COVID-19 and obstructive sleep apnoea. Sleep Med Rev. 2021;55:101382-101382.

30. Tanné F, Gagnadoux F, Chazouillères O, Fleury B, Wendum D, Lasnier E, Lebeau B, Poupon R, Serfaty L. Chronic liver injury during obstructive sleep apnea. Hepatology. 2005;41(6):1290-6.

31. Qi JC, Huang JC, Lin QC, Zhao JM, Lin X, Chen LD, Huang JF, Chen X Relationship between obstructive sleep apnea and nonalcoholic fatty liver disease in nonobese adults. Sleep Breath. 2016;20(2):529-35.

32. Minville C, Hilleret MN, Tamisier R, Aron-Wisnewsky J, Clement K, Trocme C, Borel JC, Lévy P, Zarski JP, Pépin JL. Nonalcoholic fatty liver disease, nocturnal hypoxia, and endothelial function in patients with sleep apnea. Chest. 2014;145(3):525-33.

33. Ng SS, Wong VW, Wong GL, Chu WC, Chan TO, To KW, Ko FW, Chan KP, Hui DS. CPAP did not improve nonalcoholic fatty liver disease in patients with obstructive sleep apnea: a randomized clinical trial. Am J Respir Crit Care Med. 2020;6:66

34. Norman D, Bardwell WA, Arosemena F, Nelesen R, Mills PJ, Loredo JS, Lavine JE, Dimsdale JE. Serum aminotransferase levels are associated with markers of hypoxia in patients with obstructive sleep apnea. Sleep. 2008;31(1):121-6

35. Mesarwi OA, Loomba R, Malhotra A. Obstructive sleep apnea, hypoxia, and nonalcoholic fatty liver disease. Am J Respir Crit Care Med. 2019;199(7):830-41.

36. Kendzerska T, Gershon AS, Hawker G, Leung RS, Tomlinson G. Obstructive sleep apnea and risk of cardiovascular events and all-cause mortality: a decade-long historical cohort study. PLoS Med. 2014;11(2):e1001599.

37. Cakmak E, Duksal F, Altinkaya E, Acibucu F, Dogan OT, Yonem O, Yilmaz A. Association between the severity of nocturnal hypoxia in obstructive sleep apnea and non-alcoholic fatty liver damage. Hepat Mon. 2015:15(11):e32655.

38. Buttacavoli M, Gruttad'Auria Cl, Olivo M, Virdone R, Castrogiovanni A, Mazzuca E, Marotta AM, Marrone O, Madonia S, Bonsignore MR. Liver steatosis and fibrosis in OSA patients after long-term CPAP treatment: a preliminary ultrasound study. Ultrasound Med Biol. 2016:42(1):104-9.

39. Chen LD, Zhang LJ, Lin XJ, Qi JC, Li H, Wu Z, Xu QZ, Huang YP, Lin L. Association between continuous positive airway pressure and serum aminotransferases in patients with obstructive sleep apnea. Eur Arch Otorhinolaryngol. 2018;275(2):587-94.

40. Chen LD, Huang JF, Chen QS, Lin GF, Zeng HX, Lin XF, Lin XJ, Lin L, Lin QC. Validation of fatty liver index and hepatic steatosis index for screening of non-alcoholic fatty liver disease in adults with obstructive sleep apnea hypopnea syndrome. Chin Med J (Engl). 2019;132(22):2670-6.

41. Mathiesen UL, Franzén LE, Aselius H, Resjö M, Jacobsson L, Foberg U, Frydén A, Bodemar G. Increased liver echogenicity at ultrasound examination reflects degree of steatosis but not of fibrosis in asymptomatic patients with mild/moderate abnormalities of liver transaminases. Dig Liver Dis. 2002;34(7):516-22.

42. Chuang HH, Huang CG, Chuang LP, Huang YS, Chen NH, Li HY, Fang TJ, Hsu JF, Lai HC, Chen JY, et al. Relationships among and predictive values of obesity, inflammation markers, and disease severity in pediatric 
patients with obstructive sleep apnea before and after adenotonsillectomy. J Clin Med. 2020;9(2):66.

43. Senaratna CV, English DR, Currier D, Perret J, Lowe A, Lodge C, Russell M, Sahabandu S, Matheson MC, Hamilton GS, et al. Sleep apnoea in Australian men: disease burden, co-morbidities, and correlates from the Australian longitudinal study on male health. BMC Public Health. 2016;16(Suppl 3):1029.

44. Jordan AS, McSharry DG, Malhotra A. Adult obstructive sleep apnoea. Lancet. 2014;383(9918):736-47.

45. Toyama Y, Murase K, Azuma M, Hamada S, Tachikawa R, Kubo T, Tanizawa $\mathrm{K}$, Inouch M, Handa T, Hitomi T. Impacts of long-term CPAP therapy on fatty liver in male OSA patients with abdominal obesity. Eur Respir J. 2014;44(Suppl 58):66.

46. Xu L, Lu W, Li P, Shen F, Mi YQ, Fan JG. A comparison of hepatic steatosis index, controlled attenuation parameter and ultrasound as noninvasive diagnostic tools for steatosis in chronic hepatitis B. Dig Liver Dis. 2017:49(8):910-7.

\section{Publisher's Note}

Springer Nature remains neutral with regard to jurisdictional claims in published maps and institutional affiliations.

- fast, convenient online submission

- thorough peer review by experienced researchers in your field

- rapid publication on acceptance

- support for research data, including large and complex data types

- gold Open Access which fosters wider collaboration and increased citations

- maximum visibility for your research: over $100 \mathrm{M}$ website views per year

At BMC, research is always in progress.

Learn more biomedcentral.com/submissions 